\title{
Knockdown of chemokine receptor CXCR4 gene by RNA interference: Effects on the B16-F10 melanoma growth
}

\author{
NAYARA DELGADO ANDRÉ ${ }^{1}$, VIVIANE ALINE OLIVEIRA SILVA ${ }^{2}$, \\ MARIA ANGELICA EHARA WATANABE ${ }^{3}$ and FERNANDO LUIZ DE LUCCA ${ }^{4}$
}

\begin{abstract}
${ }^{1}$ Federal University of Sao Joao del-Rei, 35501296 Divinópolis, MG; ${ }^{2}$ Molecular Oncology Research Center, Barretos Cancer Hospital, 14784-400 Barretos, SP; ${ }^{3}$ Department of Pathological Sciences, Biological Sciences Center, State University of Londrina, 86057-970 Londrina, PR; ${ }^{4}$ Department of Biochemistry and Immunology, Ribeirao Preto Medical School, University of São Paulo, 14049-900 Ribeirao Preto, SP, Brazil
\end{abstract}

Received August 13, 2015; Accepted November 4, 2015

DOI: $10.3892 / o r .2016 .4620$

\begin{abstract}
The incidence of malignant melanoma has increased greatly in recent decades presenting a high mortality rate despite intensive efforts in this area of research. Recent studies indicate that the chemokine receptor 4 (CXCR4) plays a critical role in cancer. Thus, it has been reported that CXCL12 binding to CXCR4 initiates various downstream signaling pathways that result in a plethora of responses involved in cell proliferation and metastasis. Recently, we demonstrated that CXCR4 silencing by RNA interference (RNAi) significantly reduced the number of pulmonary metastatic nodules. In the present study, we examined the effect of the intratumoral injection of CXCR4 short hairpin (shRNA) expressing plasmids on the growth of B16-F10 melanoma in mice. In vitro transfection of these tumor cells with CXCR4 shRNA expressing plasmid (CXCR4 shRNA) significantly reduced the levels of CXCR4 mRNA $(85 \%)$ and CXCR4 protein $(70 \%)$ compared with the control. We showed that the tumor growth was significantly reduced $(66 \%)$ in mice inoculated with transfected B16-F10 melanoma cells when compared with the control group. We also found that the intratumoral injection of CXCR4 shRNA expressing plasmids results in a significant inhibition (70\%) of B16-F10 melanoma growth. This finding supports the hypothesis that a direct administration of RNAi-based therapeutics into the target tumor is a promising approach for overcoming the hurdles of systemic delivery. The present study is the first demonstration that CXCR4 plays a critical role in B16-F10 melanoma growth. Currently there is great interest in the development of antagonists for therapeutic targeting CXCR4 expression. Considering our results and the fact that
\end{abstract}

Correspondence to: Dr Nayara Delgado André, Federal University of Sao Joao del-Rei, 35501296 Divinópolis, MG, Brazil

E-mail: nayara_fbq@yahoo.com.br

Key words: CXCR4, RNAi, B16-F10 melanoma, intratumoral injection, tumor growth
CXCR4 is highly conserved between human and mouse, this experimental model of cancer may be useful for the discovery of new CXCR4 antagonists with clinical implications.

\section{Introduction}

The incidence of malignant melanoma has increased greatly in recent decades presenting a high mortality rate despite intensive efforts in this area of research.

The chemokine receptor 4 (CXCR4) is a transmembrane receptor that belongs to the chemokine receptor family $\mathrm{CXC}$ which was initially reported to mediate homing of leukocytes into tissues that produce its ligand stromal cell-derived factor 1 (SDF-1), also known as CXCL12 $(1,2)$.

A growing body of evidence indicates that $\mathrm{CXCR} 4$ plays a critical role in cancer since CXCL12 binding to CXCR4 initiates various downstream signaling pathways that result in a plethora of responses involved in cell proliferation and metastasis $(3,4)$. It was reported that human melanoma cells express a high level of the chemokine receptor CXCR4 when compared with non-transformed melanocytes (5). The CXCR4 expression by malignant melanoma is predictive of poor survival rate and metastasis since its ligand CXCL12 is also increased in lungs which would explain the high frequency of pulmonary metastasis (6).

Considering the critical role of the CXCR4/CXCL12 axis in many types of cancer, including human melanoma $(7,8)$, there is currently significant interest in the discovery and development of CXCR4 antagonists (9). Therefore, there is a need of experimental models of cancer to reach this objective. One of the experimental models of melanoma research currently used is the B16 melanoma, which spontaneously originated in C57BL/6 mice. The primary tumor of B16 melanoma contains subclones which differ in their ability to form metastasis. The melanoma B16 was adapted to growth in vitro in order to compare the metastatic properties of several clones. Thus, the murine melanoma B16, F10 clone, is derived from spontaneous melanoma B16 and adapted in vitro after ten lung colonization cycles (10). Recently, we demonstrated that the knockdown of chemokine receptor CXCR4 by RNA interference (RNAi) 
significantly reduced the number of pulmonary metastatic nodules (11). However, to use this experimental model of cancer to discovery new CXCR4 antagonists, it is necessary to demonstrate that CXCR4 silencing also results in the inhibition of B16-F10 melanoma growth.

The potential therapeutic of RNAi technology has also been explored in cancer since RNAi is able to selectively knockdown critical genes involved in cell proliferation (12). The RNAi technology holds promise for cancer treatment, but many hurdles need be overcome before the clinical use of RNAi. It should be emphasized that the biggest challenge is to develop methods to specifically delivery RNAi to tumor cells. In the last few years, several strategies have been used to surmount this barrier (13-15) and the intratumoral injection of RNAi emerges as promising approach in the case of solid tumors (16). We have previously used this approach to elucidate the role played by the RNA-dependent protein kinase (PKR) in the growth and metastasis of B16-F10 melanoma (17).

In the present study, we investigated the effect of the intratumoral CXCR4 short hairpin (shRNA) expressing plasmid on the growth of the B16-F10 melanoma in C57BL/6 mice. The strategy of using a plasmid transient expression of shRNA anti-CXR4 has shown that CXCR4 plays a critical role in the initial stages of development of murine melanoma B16-F10 melanoma, suggesting that this experimental model may be useful for the discovery and development of CXCR4 antagonists.

\section{Materials and methods}

Animals. All the protocols involving animals were reviewed and approved by our Institutional Animal Care Committee. We used C57BL/6 mice, weighing 20-25 g, that were raised at the Central Animal Laboratory of the School of Medicine of Ribeirão Preto, SP, Brazil.

Culture of tumor cells. B16-F10 melanoma cells were maintained in RPMI-1640 medium supplemented with $10 \%$ inactivated fetal calf serum, $2 \mathrm{mM} \mathrm{L}$-glutamine all from (Invitrogen, Carlsbad, CA, USA) and 1\% penicillin/streptomycin (100 U/ml; Gibco Life Technologies, Carlsbad, CA, USA) in a humidified atmosphere at $37^{\circ} \mathrm{C}$ and $5 \% \mathrm{CO}_{2}$.

Target sequence selection of CXCR4 $\mathrm{mRNA}$ and plasmid vector construction. Two shRNA target sequences were selected from different positions of mouse CXCR4 cDNA sequence (GenBank, accession no. BC031665) corresponding to nucleotides 85-103 (CXCR4-1 shRNA) and 409-427 (CXCR4-2 shRNA). Each shRNA contains a sense strand of 19 nucleotides followed by a short spacer (AAGTTCTCT), the antisense strand and a stop signal (TTTTT) for RNA polymerase III.

The selection of shRNA sequences was based on the shRNA Target Finder and Design Tool available at Dharmacon website. These target sequences were submitted to a BLAST search to ensure that only the CXCR4 gene was the target. The target sequence of the negative control group named control shRNA has no homology with that of human or mice. The targeting sequence and location of each shRNA in CXCR4 cDNA are shown in Table I.
Two micrograms of sense and antisense oligonucleotides were mixed and diluted in annealing buffer to a final concentration of $40 \mathrm{ng} / \mu \mathrm{l}$. The mixture was then heated to $90^{\circ} \mathrm{C}$ for $3 \mathrm{~min}$ and then transferred to a water bath at $37^{\circ} \mathrm{C}$ and incubated for $15 \mathrm{~min}$. The annealed oligonucleotides were diluted in nuclease-free water to a final concentration of $4 \mathrm{ng} / \mu 1$. Each paired oligonucleotide was ligated overnight by enzyme T4 DNA ligase (3 U/ $\mu \mathrm{l})$ to the plasmid psiTRIKE $(50 \mathrm{ng} / \mu \mathrm{l})$. The DH5 $\alpha$ E. coli strain was used for cloning. Transformation of plasmid DNA into competent $E$. coli was performed using the heat shock method. After a short incubation in ice, a mixture of chemically competent bacteria and plasmid DNA was placed at $42^{\circ} \mathrm{C}$ for $45 \mathrm{sec}$ and then placed back in ice. LB media were added and the transformed cells were incubated at $37^{\circ} \mathrm{C}$ for $30 \mathrm{~min}$ with agitation. The $E$. coli was plated and transformed bacteria was selected based on resistance to ampicillin. Plasmid DNA was extracted of transformed $E$. coli with FlexiPrep kit (Amersham Biosciences, Little Chalfont, UK). The method employs a standard alkaline lysis procedure, including treatment with RNase and precipitation with isopropanol. Plasmid DNA was subsequently purified by Sephaglas PF resin (Amersham Biosciences) according to the manufacturer's instructions. Screening for the two inserts was performed by digestion of one microgram of plasmid DNA with PstI overnight for $37^{\circ} \mathrm{C}$.

In vitro transfection. In vitro transfection of B16-F10 melanoma cells were plated on tissue culture flasks at a density of $7 \times 10^{5}$ cells. After an overnight incubation and at a confluence $\sim 70-80 \%$, these cells were transfected with $30 \mu \mathrm{g}$ of each CXCR4 shRNA and $30 \mu \mathrm{l}$ of Lipofectamine 2000 (Invitrogen). The plasmid and Lipofectamine 2000 were diluted in serum-free medium left at room temperature for $5 \mathrm{~min}$, mixed immediately and incubated for $20 \mathrm{~min}$ at room temperature at a $\mathrm{v} / \mathrm{w}$ ratio of liposomes to shRNA of 1:1. The culture medium was removed and the shRNA-lipid complex $(1.5 \mathrm{ml}$ total volume) was added. The transfection efficiency $(\sim 75-80 \%)$ was evaluated by using the green fluorescent protein (GFP) expressing plasmid. Prior to the in vivo study, we examined whether the two plasmid-based CXCR4-specific shRNAs (CXCR4-1 shRNA and CXCR4-2 shRNA) were effective in reducing the CXCR4 expression in cultured B16-F10 cells. Thus, tumor cells were transfected with CXCR4-1, CXCR4-2 or control shRNA for $5 \mathrm{~h}$ and thereafter the cells were washed, suspended in medium and maintained in culture for 24 or $48 \mathrm{~h}$. To determine the amount of mRNA CXCR4 and CXCR4 protein, lysates of the B16-F10 melanoma cells were used for RNA isolation and western blot analysis.

RNA isolation. Total cellular RNA was extracted using TRIzol-LS Reagent (Invitrogen). The integrity of RNA was assessed using the Bioanalyzer (Agilent Technologies, Santa Clara, CA, USA).

Analysis of CXCR4 expression. Reverse transcription-PCR was performed with $1.2 \mu \mathrm{g}$ of the isolated total RNA and synthesized to cDNA in a $25 \mu 1$ reaction system using reverse transcriptase (Promega, Madison, WI, USA). RT conditions were $5 \mathrm{~min}$ denaturation at $65^{\circ} \mathrm{C}, 60 \mathrm{~min}$ at $37^{\circ} \mathrm{C}$ and $5 \mathrm{~min}$ at $75^{\circ} \mathrm{C}$ in a thermocycler (Abgene, Epsom, UK). Reverse 
Table I. Sequences of shRNA oligonucleotides.

\begin{tabular}{lcl}
\hline shRNA & $\begin{array}{c}\text { Target } \\
\text { Position }^{\mathrm{a}}\end{array}$ & \\
\hline CXCR4-1 & $85-103$ & F: 5'-ACCGCGATCAGTGTGAGTATATAAAGTTCTCTTATATACTCACGATCGCTTTTC-3' \\
& & R: 5'-GCAGAAAAAGCGATCAGTGTGAGTATATAAGAGAACTTTATATACTCACACTGATCG-3' \\
CXCR4-2 & $409-427$ & F: 5'-ACCGGTAAGGCTGTCCATATCATAAGTTCTCTATGATATGGACAGCCTTACCTTTTC-3' \\
& & R: 5'-GCAGAAAAAGGTAAGGCTGTCCATATCATAGAGAACTTATGATATGGACAGCCTTACCGGT-3' \\
Control & & F: 5'-ACCGAAGCGCTGCCGCGACGTTGAAGTCTCTCAACGTCGCGGCAGCGCTTCTTTTC-3' \\
& R: 5'-TGCAGAAAAAGAAGCGCTGCCGCGACGTTGAGAGAACTTCAACGTCGCGGCAGCGCTTCGGT-3'
\end{tabular}

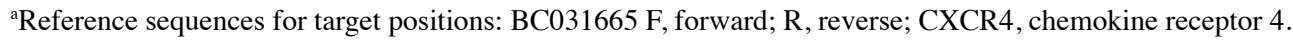

Table II. Polymerase chain reaction primer sequences.

\begin{tabular}{lll}
\hline Genes & \multicolumn{1}{c}{$\begin{array}{c}\text { Primer } \\
\text { sequences }\end{array}$} & $\begin{array}{c}\text { GenBank } \\
\text { acession no. }\end{array}$ \\
\hline CXCR4 & F: 5'-ACAGGTACATCTGTGACCGCCTTT-3' & BC031665 \\
& R: 5'-TGCTCTCGAAGTCACATCCTTGCT-3' & \\
$\beta$-actin & F: 5'-TGGAATCCTGTGGCATCCATGAAAC-3' & BC014861 \\
& R: 5'-TAAAACGCAGCTCAGTAACAGTCCG-3' & \\
\hline
\end{tabular}

F, forward; R, reverse; CXCR4, chemokine receptor 4.

transcription was carried out with $0.5 \mu \mathrm{g}$ of the oligodT primer, 1 unit of reverse transcriptase, 1 unit of RNase inhibitor (all from Invitrogen), $5 \mu \mathrm{l}$ of $5 \mathrm{X}$ buffer and $4 \mu \mathrm{l} \mathrm{MgCl}_{2}$. The $\beta$-actin mRNA was used as a loading control. PCR conditions for $\beta$-actin were $4 \mathrm{~min}$ denaturation at $94^{\circ} \mathrm{C}, 40$ cycles of $1 \mathrm{~min}$ at $94^{\circ} \mathrm{C}, 1 \mathrm{~min}$ at $52^{\circ} \mathrm{C}$ and $2 \mathrm{~min}$ at $72^{\circ} \mathrm{C}$ and $10 \mathrm{~min}$ elongation at $72^{\circ} \mathrm{C}$ in a thermocycler (Abgene). PCR conditions for CXCR4 were 5 min denaturation at $94^{\circ} \mathrm{C}, 35$ cycles of $1 \mathrm{~min}$ at $94^{\circ} \mathrm{C}$, $1 \mathrm{~min}$ at $51^{\circ} \mathrm{C}$ and $1 \mathrm{~min}$ at $72^{\circ} \mathrm{C}$, and $10 \mathrm{~min}$ elongation at $72^{\circ} \mathrm{C}$ in a thermocycler (Abgene). The primers sequences and GenBank Accession number are shown in Table II. PCR products of $\beta$-actin (364 bp) and CXCR4 (291 bp) were analysed by electrophoresis in a $1.5 \%$ agarose gel and visualized using UV fluorescence after staining with ethidium bromide. Quantification of CXCR4 bands was performed by using ImageQuant software, version 3.3 (Molecular Dynamics, Inc., Sunnyvale, CA, USA) and the results were expressed in terms of percentage.

Western blot analysis. B16-F10 adherent cells were detached using EDTA with RPMI without fetal bovine serum and centrifuged at 4,000 rpm for $15 \mathrm{~min}$. The cell pellet was resuspended in $300 \mu \mathrm{l}$ PBS plus the proteases inhibitors $0.1 \%$ aprotinin, $0.1 \%$ leupepsin and $1 \%$ Triton. The sample was incubated under agitation on ice for $20 \mathrm{~min}$ and after centrifuged at 12,000 rpm for $15 \mathrm{~min}$ at $4^{\circ} \mathrm{C}$ and the protein concentration determined by Cadman method. Total cellular protein $(30 \mu \mathrm{g})$ was separated by electrophoresis through a $10 \%$ SDS-PAGE resolving gel with an SDS-PAGE stacking gel. After electrophoresis, proteins were transferred onto a Hybond-C supported nitrocellulose membrane (Amersham Biosciences) by electroblotting for $4 \mathrm{~h}$ at $45 \mathrm{~V}, 25^{\circ} \mathrm{C}$, in transfer buffer $(3.94 \mathrm{~g}$ Tris- $\mathrm{HCl}$, $18.80 \mathrm{~g}$ glycine, $240 \mathrm{ml}$ methanol, $10 \% \mathrm{SDS})$. The membrane was then blocked with $10 \%$ dried milk in TBS (20 mM Tris, $500 \mathrm{mM} \mathrm{NaCl}$ ) at room temperature overnight, after washed twice followed by incubation at room temperature with 1:250 off rabbit anti-CXCR4 polyclonal antibody (Santa Cruz Biotechnology, Santa Cruz, CA, USA) in TBS-Tween-20 buffer for $90 \mathrm{~min}$. The membrane was washed in TBS 1X Tween-20 for $30 \mathrm{~min}$ and secondary anti-rabbit antibodies labeled with horseradish peroxidase (Amersham Biosciences) was added and the membrane incubated at room temperature for $60 \mathrm{~min}$ under agitation. Membranes were washed twice in TBS-T for $20 \mathrm{~min}$ and in TBS for $5 \mathrm{~min}$. Antibody labeled protein bands were visualized with ECL detection reagents (Amersham Biosciences) applied following the manufacturer's protocol. To use $\beta$-actin as a loading control, a second gel was loaded with identical volume of the experimental sample followed by blotting with the anti- $\beta$-actin antibody and the detection was performed as described for CXCR4. Quantification of bands was performed by using Image Quant software, version 3.3 (Molecular Dynamics Inc.) and the results were expressed in terms of percentage.

Tumorigenic assay. Based on the in vitro findings, CXCR4-1 shRNA was selected for in vivo experiments. B16-F10 melanoma cells were transfected with CXCR4-1 shRNA or control shRNA for $5 \mathrm{~h}$ and thereafter the cells were washed, suspended in medium and maintained in culture for $24 \mathrm{~h}$ to inject into mice. After this treatment, tumor cells were detached with EDTA, washed twice in PBS and finally resuspended in RPMI. The viability of cells was assessed by trypan blue staining and was $>95 \%$. Tumor cells transfected were then inoculated subcutaneously $\left(2 \times 10^{5}\right.$ cells/animal) into the right flank of mice ( $n=10$ per group), animals were euthanized 14 days after treatment according to Kumar et al (18) and tumors were excised and weighted on a microbalance Sartorius Supermicro (model S4; Sartorius, Goettingen, Germany).

Intratumoral injection of CXCR4-1 shRNA. Each animal received $2 \times 10^{5}$ tumor cells subcutaneously into the right flank ( $n=10$ per group). The tumors were monitored, and treatment began when the average tumor diameter reached 5-7 mm, typically 7 days after of tumor cell inoculation. This tumor size allows the intratumoral injection to be performed with 


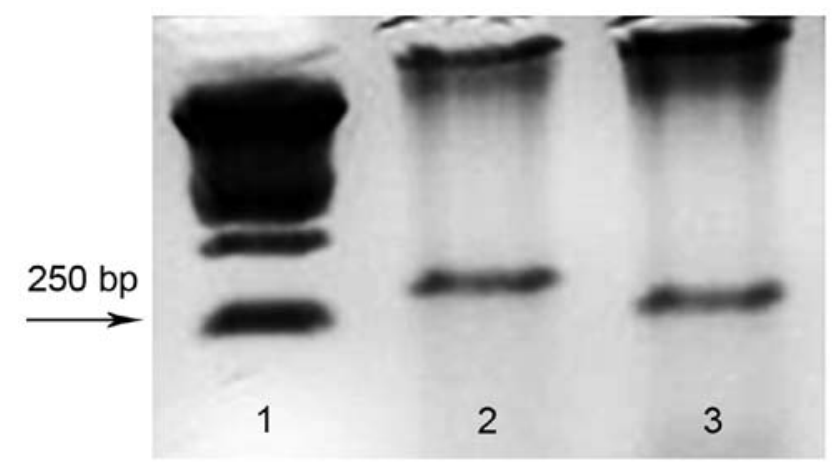

Figure 1. Analysis of CXCR4 expression in B16-F10 melanoma cells by electrophoresis on agarose gels. The experimental conditions used in RT-PCR and electrophoresis on agarose were as described in Material and methods. Lane 1 DNA ladder (250 bp) used as marker of molecular weight, lane 2, $\beta$-actin (364 bp) and lane 3, CXCR4 (291 bp).

safety. Thus, after 7 days of tumor inoculation mice received a single intratumoral injection of $2 \mu \mathrm{g}$ of CXCR4-1 shRNA complexed with $2 \mu \mathrm{l}$ of Lipofectamine 2000 dissolved in $50 \mu \mathrm{l}$ of RPMI. Some mice received an intratumoral injection of $2 \mu \mathrm{g}$ control shRNA-expressing plasmid complexed with $2 \mu \mathrm{l}$ of Lipofectamine 2000 as a negative control. The mice were sacrificed 7 days after the injection and the tumors weighed.

Statistical analysis. One-way analysis of variance (ANOVA) was used to analyze the significance between groups. All data represent mean $\pm \mathrm{SD}$. $\mathrm{P}<0.05$ was considered to indicate a statistically significant difference.

\section{Results}

Analysis of CXCR4 expression by B16-F10 melanoma cells. Several studies have demonstrated the involvement of chemokines and their receptors in tumor growth and metastasis. In order to investigate the role of CXCR4 chemokine receptor in a model of murine melanoma, we first examined whether B16-F10 melanoma cells express this chemokine receptor. Our results indicate that these tumor cells constitutively express CXCR4 as shown in Fig. 1.

Knockdown of CXCR4 mRNA by transfection of B16-F10 melanoma cells with CXCR4 shRNA. To reduce the expression of CXCR4 mRNA in B16-F10 melanoma cells, two CXCR4 shRNAs were designed. Each CXCR4 shRNA was annealed and ligated into the psiSTRIKE vector controlled by Pol III U6 promoter. The tumor cells were transfected for 24 and $48 \mathrm{~h}$ with the plasmid-based CXCR4-1 shRNA, CXCR4-2 shRNA or control shRNA expressing plasmids with Lipofectamine 2000. After transfection, CXCR4 mRNA degradation was monitored by RT-PCR. Fig. 2A shows that only the plasmid-based CXCR4-1 shRNA significantly reduced the level of CXCR4 mRNA $(85 \%, \mathrm{P}<0.001)$ after $48 \mathrm{~h}$ of transfection and this effect remains for up to 4 days (data not shown).

Reduction of CXCR4 protein level by transfection of B16 melanoma cells with CXCR4 shRNA. The level of CXCR4 protein in B16-F10 melanoma cells transfected with CXCR4-1 shRNA, CXCR4-2 shRNA or control shRNA was evaluated by
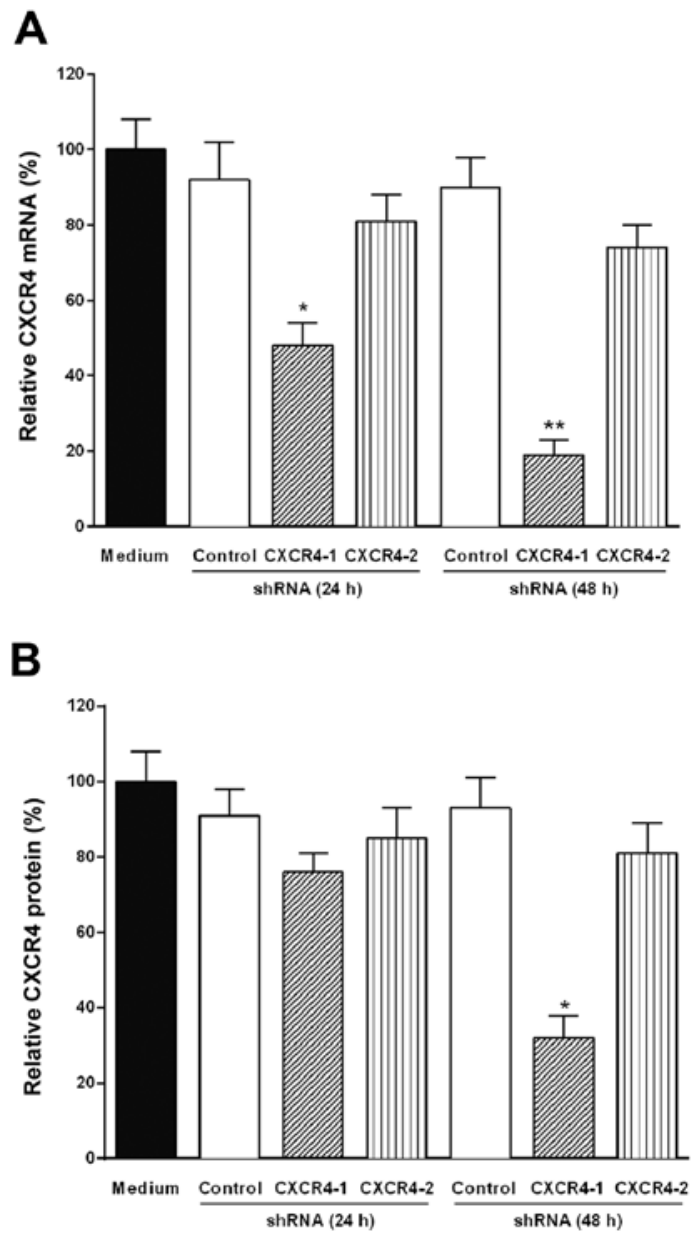

Figure 2. Level of CXCR4 mRNA and CXCR4 protein in B16-F10 melanoma cells transfected with CXCR4 shRNA. (A) CXCR4 mRNA expression after transfection of B16-F10 melanoma cells for 24 or $48 \mathrm{~h}$ with CXCR4-1 shRNA, CXCR4-2 shRNA or control shRNA; (B) CXCR4 protein after transfection of B16-F10 melanoma cells for 24 or $48 \mathrm{~h}$ with CXCR4-1 shRNA, CXCR4-2 shRNA or control shRNA. The $\beta$-actin was used as a loading control. The level of CXCR mRNA and CXCR4 protein in B16-F10 melanoma cells incubated with RPMI medium was set as $100 \%$. Results are expressed as the means \pm SD of three independent experiments and the bands are representative of one typical experiment. ${ }^{*} \mathrm{P}<0.001$.

western blot analysis. The downregulation of CXCR4 protein expression was also significantly $(70 \%, \mathrm{P}<0.001)$ observed after $48 \mathrm{~h}$ of transfection with CXCR4-1 shRNA as shown in Fig. 2B.

Transfection of B16-10 melanoma cells with CXCR4 shRNA inhibits tumor growth in mice. The B16-F10 melanoma cells transfected with CXCR4-1 shRNA or control shRNA were injected subcutaneously into C57BL/6 mice. The animals were sacrificed 14 days after the inoculation of B16-F10 cells and tumors were excised and weighted. Fig. 3 shows that tumor growth was significantly inhibited $(66 \%, \mathrm{P}<0.001)$ when B16-F10 melanoma cells were transfected with CXCR4-1 shRNA when compared to control shRNA.

Intratumoral injection of the CXCR4-1 shRNA-expressing plasmid inhibits B16-F10 melanoma growth. To investigate the effect of the intratumoral injection of the CXCR4-1 shRNA-expressing plasmids on melanoma growth, mice 


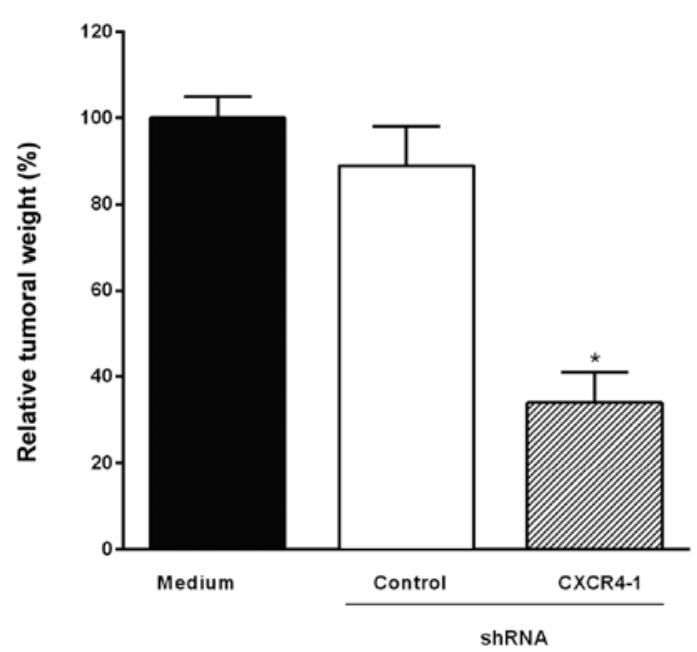

Figure 3. Effect of CXCR4 silencing on B16-F10 melanoma growth in mice. Animals were subcutaneously injected with B16-F10 melanoma cells transfected with CXCR4-1 or control shRNA. Mice were used in groups of 10 . Animals were sacrificed after 14 days and tumors were excised and weighed. Results are expressed as the mean $\pm \mathrm{SD}$ and the mean of the tumor weight corresponding to control group was set as $100 \%$. Data of one representative experiment of three are shown. ${ }^{*} \mathrm{P}<0.001$.

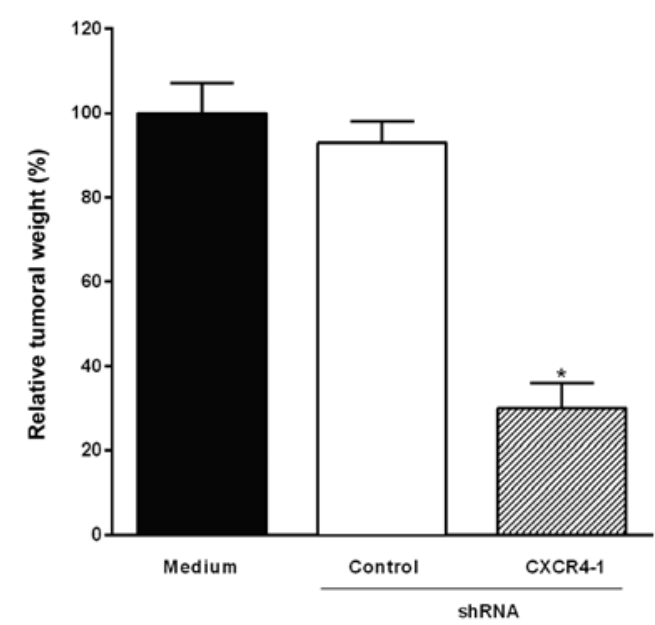

Figure 4. Effect of intratumoral injection of CXCR4 shRNA or control shRNA on tumor growth in C57BL/6 mice. B16-F10 melanoma cells were subcutaneously inoculated into C57BL/6 mice ( $\mathrm{n}=10$ per group). After 7 days of tumor cell inoculation, the mice received a single intratumoral injection of CXCR4-1 shRNA or control shRNA. Mice were sacrificed 7 days after injection, tumors were removed and weighed. Results are expressed as the means $\pm \mathrm{SD}$ and the mean of the tumor weight corresponding to control group was set as $100 \%$. Data of one representative experiment of three are shown. ${ }^{*} \mathrm{P}<0.001$.

were inoculated subcutaneously with B16-F10 melanoma cells. Fig. 4 shows a significant reduction of tumor weight $(70 \%, \mathrm{P}<0.001)$ in mice that had received a single intratumoral injection of the CXCR4-1 shRNA compared to animals injected with the plasmid-based control shRNA.

\section{Discussion}

Current strategies for studying the involvement of CXCR4 in cancer are based on receptor blockade in the surface of tumor cells with specific antagonists or antibodies. In the present

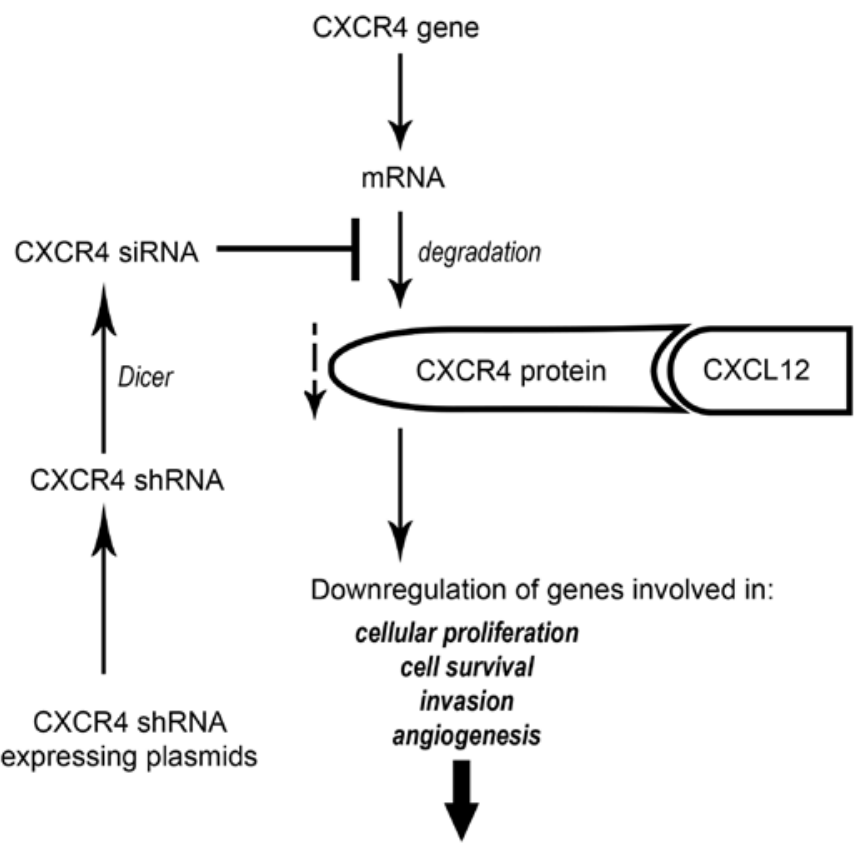

INHIBITION OF TUMOR GROWTH

Figure 5. Overview of proposed molecular mechanisms involved in the inhibition of B16-F10 melanoma growth after CXCR4 silencing with CXCR4 shRNA expressing plasmids. CXCR4, chemokine receptor 4; CXCL12, ligand of CXCR4; shRNA, short hairpin RNA; siRNA, small interfering RNA.

study, we applied the RNAi technology to elucidate the role played by CXCR4 in B16-F10 melanoma growth. The first step was to examine the ability of two CXCR4-specific shRNAs expressing plasmids to downregulate CXCR4 mRNA and CXCR4 protein in vitro. Our results indicate that B16-F10 melanoma cells constitutively express CXCR4 and that only CXCR4-1 shRNA significantly reduced the level of CXCR4 mRNA and CXCR4 protein after in vitro transfection of tumor cells. Thus, CXCR4-1 shRNA was used in all further experiments. The next step was to investigate the effect of silencing CXCR4 expression on B16-F10 melanoma growth in vivo. To address this question, the transfected tumor cells with CXCR4-1 shRNA or control shRNA in vitro were injected subcutaneously into mice. Our findings showed that the tumor growth was significantly reduced only in mice inoculated with B16-F10 melanoma cells transfected with CXCR4-1 shRNA, suggesting that CXCR4 plays a critical role in B16-F10 melanoma growth.

It is known that RNAi-based therapy is effective and elicit gene silencing response, the double-stranded RNA molecules must be delivered to the target cells. Unfortunately, the specific delivery of RNAi has been challenging despite many efforts made in the last few years and the intratumoral injection of RNAi has emerged as a promising alternative to overcome this obstacle $(19,20)$. We also decided to investigate the effect of the intratumoral injection of CXCR4 shRNA expressing plasmid in the pre-established subcutaneous B16-F10 melanoma. Our results indicate that the intratumoral injection of CXCR4-1 shRNA significantly inhibited tumor growth when compared to animals injected with the plasmid-based control shRNA. It is worthwhile that this effect was obtained with 
only a single injection of the CXCR4-1 shRNA expressing plasmid and this approach for RNAi delivery was effective for at least a week.

The role of the CXCR4/CXCL12 axis in cancer has been extensively investigated in the last decade. Thus, it was found that CXCR4 is overexpressed in $>23$ human cancers, including melanoma and contributes to cell proliferation, cell survival, invasion ad angiogenesis (21).

Based on these findings, we proposed that the effect of CXCR4 shRNA expressing plasmids on B16-F10 melanoma growth could be explained as illustrated in Fig. 5. Briefly, CXCR4 shRNA expressing plasmids were used to transfect the B16-F10 melanoma cells in vitro or in vivo by intratumoral injection. After the uptake of CXCR4 shRNA expressing plasmids by tumor cells, CXCR4 shRNA are transcribed in the nucleus, exported to the cytoplasm and processed by Dicer to generate CXCR4 siRNA which induces the specific degradation of CXCR4 mRNA. Therefore, the level of CXCR4 protein is decreased with subsequent downregulation of genes involved in cell survival, cell adhesion, invasion and angiogenesis, resulting in the inhibition of B16-F10 melanoma growth.

The present study gives support to the concept that a direct administration of RNAi-based therapeutics into the target tumor is a promising approach for overcoming the hurdles of systemic delivery. Our findings also suggest that the intratumoral injection of CXCR4-1 shRNA expressing vector may be a novel therapeutic approach for human solid tumors such as cutaneous melanoma and breast cancer since CXCR4 is overexpressed in these tumors.

It should be emphasized that the present study is the first demonstration that CXCR4 plays a critical role in the growth of B16-F10 melanoma. However, further work is required to elucidate the molecular mechanisms involved in this phenomenon. Currently there is great interest in the discovery of antagonists for therapeutic targeting CXCR4 expression. Considering that our results indicate that CXCR4 is implicated in the early stages of B16-F10 melanoma growth, its role well established in metastasis and the fact that this chemokine receptor is highly conserved between human and mouse (22), this experimental model of cancer may contribute for the discovery of CXCR4 antagonists with clinical implications.

\section{Acknowledgements}

The present study was supported by FAPESP (06/57963-1) . We thank Cacilda D. Pereira and Zuleica A. S. Moraes for the technical assistance.

\section{References}

1. Murdoch C: CXCR4: Chemokine receptor extraordinaire. Immunol Rev 177: 175-184, 2000.

2. Zlotnik A: Chemokines in neoplastic progression. Semin Cancer Biol 14: 181-185, 2004.
3. Toyozawa S, Kaminaka C, Furukawa F, Nakamura Y, Matsunaka $\mathrm{H}$ and Yamamoto Y: Chemokine receptor CXCR4 is a novel marker for the progression of cutaneous malignant melanomas. Acta Histochem Cytochem 45: 293-299, 2012.

4. Mitchell B, Leone D, Feller JK, Bondzie P, Yang S, Park HY and Mahalingam M: Correlation of chemokine receptor CXCR4 mRNA in primary cutaneous melanoma with established histopathologic prognosticators and the BRAF status. Melanoma Res 24: 621-625, 2014.

5. Payne AS and Cornelius LA: The role of chemokines in melanoma tumor growth and metastasis. J Invest Dermatol 118: 915-922, 2002

6. Longo-Imedio MI, Longo N, Treviño I, Lázaro $\mathrm{P}$ and Sánchez-Mateos P: Clinical significance of CXCR3 and CXCR4 expression in primary melanoma. Int J Cancer 117: 861-865, 2005.

7. Scala S, Ottaiano A, Ascierto PA, Cavalli M, Simeone E, Giuliano P, Napolitano M, Franco R, Botti G and Castello G: Expression of CXCR4 predicts poor prognosis in patients with malignant melanoma. Clin Cancer Res 11: 1835-1841, 2005.

8. ScalaS, GiulianoP, AsciertoPA, Ieranò C, FrancoR, Napolitano M, Ottaiano A, Lombardi ML, Luongo M, Simeone E, et al: Human melanoma metastases express functional CXCR4. Clin Cancer Res 12: 2427-2433, 2006.

9. Duda DG, Kozin SV, Kirkpatrick ND, Xu L, Fukumura D and Jain RK: CXCL12 (SDF1 $\alpha$ )-CXCR4/CXCR7 pathway inhibition: An emerging sensitizer for anticancer therapies? Clin Cancer Res 17: 2074-2080, 2011.

10. Fidler IJ: Biological behavior of malignant melanoma cells correlated to their survival in vivo. Cancer Res 35: 218-224, 1975.

11. André ND, Silva VAO, Ariza CB, Watanabe MAE and De Lucca FL: In vivo silencing of CXCR4 with jetPEI/CXCR4 shRNA nanoparticles inhibits pulmonary metastasis of B16-F10 melanoma cells. Mol Med Rep 12: 8320-8326, 2015.

12. Li Z, Li N, Wu M, Li X, Luo Z and Wang X: Expression of miR-126 suppresses migration and invasion of colon cancer cells by targeting CXCR4. Mol Cell Biochem 381: 233-242, 2013.

13. Díaz MR and Vivas-Mejia PE: Nanoparticles as drug delivery systems in cancer medicine: Emphasis on RNAi-containing nanoliposomes. Pharmaceuticals (Basel) 6: 1361-1380, 2013.

14. Li CX, Parker A, Menocal E, Xiang S, Borodyansky L and Fruehauf JH: Delivery of RNA interference. Cell Cycle 5: 2103-2109, 2006.

15. Sakurai Y, Hatakeyama H, Sato Y, Hyodo M, Akita H and Harashima H: Gene silencing via RNAi and siRNA quantification in tumor tissue using MEND, a liposomal siRNA delivery system. Mol Ther 21: 1195-1203, 2013.

16. Deharvengt SJ, Gunn JR, Pickett SB and Korc M: Intratumoral delivery of shRNA targeting cyclin D1 attenuates pancreatic cancer growth. Cancer Gene Ther 17: 325-333, 2010.

17. André ND, Silva VAO, Watanabe MAE and De Lucca FL: Intratumoral injection of PKR shRNA expressing plasmid inhibits B16-F10 melanoma growth. Oncol Rep 32: 2267-2273, 2014.

18. Kumar R, Yoneda J, Fidler IJ and Dong Z: GM-CSF-transduced B16 melanoma cells are highly susceptible to lysis by normal murine macrophages and poorly tumorigenic in immune-compromised mice. J Leukoc Biol 65: 102-108, 1999.

19. Deng Y, Wang CC, Choy KW, Du Q, Chen J, Wang Q, Li L, Chung TK and Tang T: Therapeutic potentials of gene silencing by RNA interference: Principles, challenges, and new strategies. Gene 538: 217-227, 2014.

20. Li T, Wu M, Zhu YY, Chen J and Chen L: Development of RNA interference-based therapeutics and application of multi-target small interfering RNAs. Nucleic Acid Ther 24: 302-312, 2014.

21. Chatterjee S, Behnam Azad B and Nimmagadda S: The intricate role of CXCR4 in cancer. Adv Cancer Res 124: 31-82, 2014.

22. Heesen M, Berman MA, Benson JD, Gerard C and Dorf ME: Cloning of the mouse fusin gene, homologue to a human HIV-1 co-factor. J Immunol 157: 5455-5460, 1996. 